\title{
Depression Predictor Model for Farmers using Machine Learning Techniques
}

\author{
Dr. Mallikarjun H M, Akshay Chhetri, Apoorva G S, Gowri Jadhav, Sheetal B V
}

\begin{abstract}
There are a few disorders that are the outcome of unbalanced mental state. A very basic one is depression. Depression is a very serious yet common mental ailment that damagingly distresses how a person thinks or feels or acts.

Side effects of physical injuries are obvious and regularly agonizing, because of which they are recognized and paid attention to. Symptoms of mental illnesses are not very comprehendible. A lot of individuals don't know about them, including the people who are suffering.

This research paper proposes a methodology with an approach to machine learning in order to categorize the subject into 4 distinguished levels of depression, namely normal, mildly depressed, moderately depressed and severely depressed. This procedure is proposed to be carried out using PHQ-9 and DASS-21 questionnaire and the electric EEG bands Alpha, Beta, Delta, Gamma and Theta variations will be obtained via the usage of head kit Neurosky's Mindwave aid.
\end{abstract}

\section{Keywords: PHQ-9, DASS-21, EEG, SVM}

\section{INTRODUCTION}

\section{A. Depression}

Depression is a very serious yet common mental ailment that damagingly distresses how a person thinks or feels or acts. Nonetheless, it is remediable if diagnosed at an earlier stage. Depression leads to diminishing interest in day-to-day happenings, which leads to significant hindrance in everyday life. It can also lead to physical and mental stress and can weaken subject's ability to work.

Some of the features can play an important part in depression like changes in certain chemicals existing in the brain might lead to symptoms of depression, depression can likewise run in families. To take an example, if one identical twin suffers from depression, then the other one has a chance of 70 percent of suffering from the same illness sometime in future. People with low self-worth, are easily overcome by stress, or who are generally negative are more likely to suffer from depression. Even continuous exposure to negligence, violence, exploitation or poverty may lead to people being vulnerable to depression.

Revised Manuscript Received on December 15, 2019.

Dr. Mallikarjun H M department of Electronic \& Instrumentation Engg, RNSIT, Bengaluru.

Akshay Chhetri, BE in Electronics and Instrumentation Engg. RNSIT, Bengaluru

Apoorva G S, BE in Electronics and Instrumentation Engg. RNSIT, Bengaluru

Gowri Jadhav, BE in Electronics and Instrumentation Engg. RNSIT, Bengaluru

Sheetal B V, BE in Electronics and Instrumentation Engg. RNSIT, Bengaluru

\section{B. Electroencephalogram}

Most of the diagnostic tests that are carried out on the brain showcase structural differences that can cause changes in behavior. Any structural variations such as presence of tumors will be focused in an MRI or X-ray. But an Electroencephalogram (EEG) provides information about the actual working of the brain.

EEG is a technique which helps in recording the electrical activity of human's brain. This method is non-invasive and it involves placement of electrodes on the patient's scalp. Acquisition method: EEG signal is obtained by measuring the voltage fluctuations inside the neurons of brains. It can be obtained by using the 10/20 placement of electrodes on the scalp. The electrodes are placed in a predefined area and the signals are then obtained from these areas. Alpha, beta, gamma, theta and delta are known as rhythms and are obtained from electrode placement sections of the scalp.

\section{Farmers and Depression}

Farmer suicides have ended up being one of the significant policy worry in India which has brought about significant impact on the quality of farmers life. As indicated by the United Nations Commission on Sustainable Development (UNCSD), one farmer committed suicide at an interval of every 32 minute between 1997 and 2005 in India. The likelihood of farmers turning to the severe suicidal step was disturbing in Maharashtra, Karnataka, Telangana, Chhattisgarh and MP during 2014 according to National Crime Records Bureau (NCRB). Amongst the total states, $90 \%$ of farmer suicides (5056) are accounted by these states. The tragic occurrences of farmer suicides proceeded in the ensuing years. In comparison to 2014. the number of farmers committing suicide shot up by 42 per cent in 2015 . The five states continued to exist as suicides hotspots in both the years because of some familiar farming conditions along with the history of agricultural growth in these states.

Decrease in the rate of farmers committing suicide was noted only in the state of Chhattisgarh when compared among the 5 states in between the year 2014 and 2015. It is crucial to observe that $50 \%$ of the farmer suicides revealed in the year 2014 and 2015 happened during the term between July to November. During 2014, 15 farmers per day committed suicide in India and by 2015 this reached up to 21 per day. Numerous studies have tended to the subject of farmer suicides from different viewpoints. A comprehensive review of the publications in India proposes numerous reasons and these could be put together into one, namely rising distress in the farming zone.

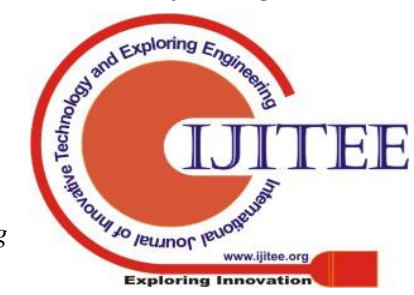


Low productivity, climatic reasons, and market failure further aggravates these distresses in both the factor as well as product markets.

Karnataka's Farmers have been facing several issues ranging from improper technology to agricultural loan debts. Karnataka is a vast state with varied topography, while there are agriculturally prosperous regions; some regions are severely affected by the frequent droughts.

The Times of India (28 September 2018) stated that between April 1 and august 31, 2018 at least one farmer committed suicide every 12 hours in Karnataka. According to the agricultural department, 283 farmer suicides were reported.

Four farmer suicides for each day was recorded by the Department of Agriculture between the term 2015- July to 2016- June in Karnataka. Out of 1490 farmer suicides reported during this term in Karnataka, 80 percent were committed by small farmers. The time between 2015- July to 2015- October was a pinnacle of farmers disappointment in Karnataka. 56 percent of all suicides took place during this term. The severity of suicides in the district of MP was incredibly high at 62 fatalities for each lakh hectares of net cultivated area and 49 farmer suicides for every lakh hectare of gross cultivated area. Haveri had the extreme number of 57 fatalities for each lakh of cultivating relatives.

\section{LITERATURE SURVEY}

The authors [1] have put light on the usage of EEG signals for investigation of depression. Grouping of affected patients from ordinary subjects was done by utilizing EEG signals and techniques like Machine Learning, FFT algorithms and other signal processing techniques. This will thus help psychiatrists to gauge the extent of depression in patients.

The authors [2] in this paper recorded EEG signals from the members by utilizing their facial stimuli using high gamma and low gamma rays. It was found out that the coherence of the right part of the brain under normal circumstances was more than the findings of the same in moderately depressed patients. The final results obtained by the authors [2] show more cooperation in the right parts of the brain in normal conditions as opposed to the findings of the same in the moderately depressed group. The results of the prefrontal and parietal regions of the moderately depressed patients were less under normal conditions.

The author [3] has described in detail about the statistics of suicides of farmers in India as well as its impacts and actions taken by the government in respect to this. Suicides by farmers represent $11.3 \%$ of the total suicides in India. There are numerous reasons leading to their suicide such as high obligation troubles, genetically altered harvests, policies by the government, individual issues and family issues. The National Crime Records Bureau of India, in 2012 reported 13,754 farmer suicides. The highest number of farmer suicides was reported in 2004 where the number rose to 18,241 .

The authors[4] in their work had proposed steps towards the development of a particular type of approach where the selection of features, experiments based on fusion and classification were undertaken to infer the behavioral types and combinations which could best distinguish between the depressed and non-depressed subjects. With the help of statistics, features were extracted from the head orientation, activity of the eye and speaking. Characterization of behavioral traits of major depression was done along with examination of the routine of the distinct modalities when combined.

The authors[5] in this letter have aimed towards the development of a model which would be helpful in the prediction of anxiety and depression of the older subjects using ML technology. The data of 510 old patients were evaluated and tested. An all-time high accuracy of $89 \%$ using the random forest classifier method was obtained. This model was compared and tested with another set of similar data to validate it. Predictive precision was around $91 \%$ and the false positive rate was around $10 \%$. This comparison was done using the gold standard tool.

The authors [6] in this project acquired the Electroencephalogram (EEG) signal from the accessible public database and processed them using MATLAB. This would be of greater significance in the classification of patients with disorders utilizing classifier devices. For this, the extraction of various features were done form frequencies namely, alpha, delta and theta.

The authors[7] of this paper have presented a technique involving Machine Learning to classify the depressed patients into four bands of depression, each significant of a particular level of the same according to the Beck depression inventory score. Along with the above method, a FFNN (Fuzzy Functions based on Neural Networks) classifier was proposed. The dataset consisted of 60 records of EEG signals of different levels of the depressed patients in the rest state using features which were not linear along with KFD(Katz Fractal Dimension) and FFD(Fuzzy Fractal Dimension). The outcomes showed that Katz Fractal Dimension has better accuracy than the other techniques of prediction, especially SVM (Support Vector Machine).

The authors [8] in this paper have depicted a technique for combining both EEG signal analysis and facial emotion recognition through video analysis to viably sort depression into different levels. In order to achieve this, the spectrum of 3 frequency bands and all the bands of EEG are utilized as highlights alongside standard deviation, mean and entropy.

The authors [9] in their work have presented a technique wherein the Becks index of each patient is estimated by the removal of select features from the subjects EEG signal. In order to complete the quantification of depression, an algorithm had been constructed which utilizes the readings received from the fuzzy classifier and the SVM(Support Vector Machine). From this methodology, the desirable outcomes were acquired showing that the algorithm proposed had a decent capacity to decide the record for depression. The outcomes acquired had a small percent relative difference of around 5\% and the Pearson correlation of around 0.92. The outcomes indicated that the estimation with which the system was designed had a high correlation and a low PRD amount when compared to the original Beck number in relation to 
each subject.

After reviewing the literatures it's found that very less researchers succeeded to predict depression disorders in various levels. Most of the works ended with limited EEG data samples of Indian farmers, therefore, with the help of high number of data samples and utilization of advanced machine learning algorithms in the said work, the scope can be improved.

\section{RESULTS AND DISCUSSIONS}

EEG signals are helpful in the investigation of depression state. It gives a high exactness than different signals for depression examination. The reason for the proposed architecture is to guarantee that effectiveness and precision will be increased. Proposed Block Diagram as shown in Fig 1.

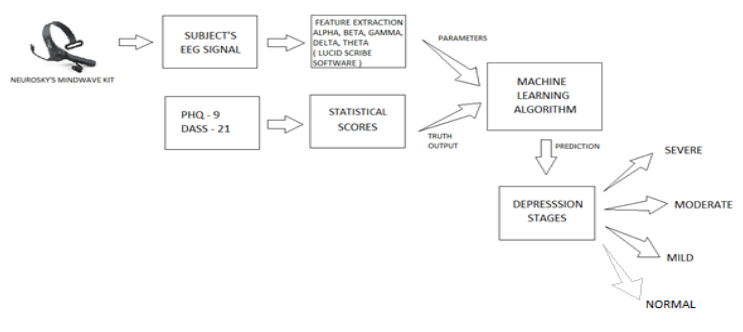

Fig. 1.Proposed Block diagram of Depression Predictior Model
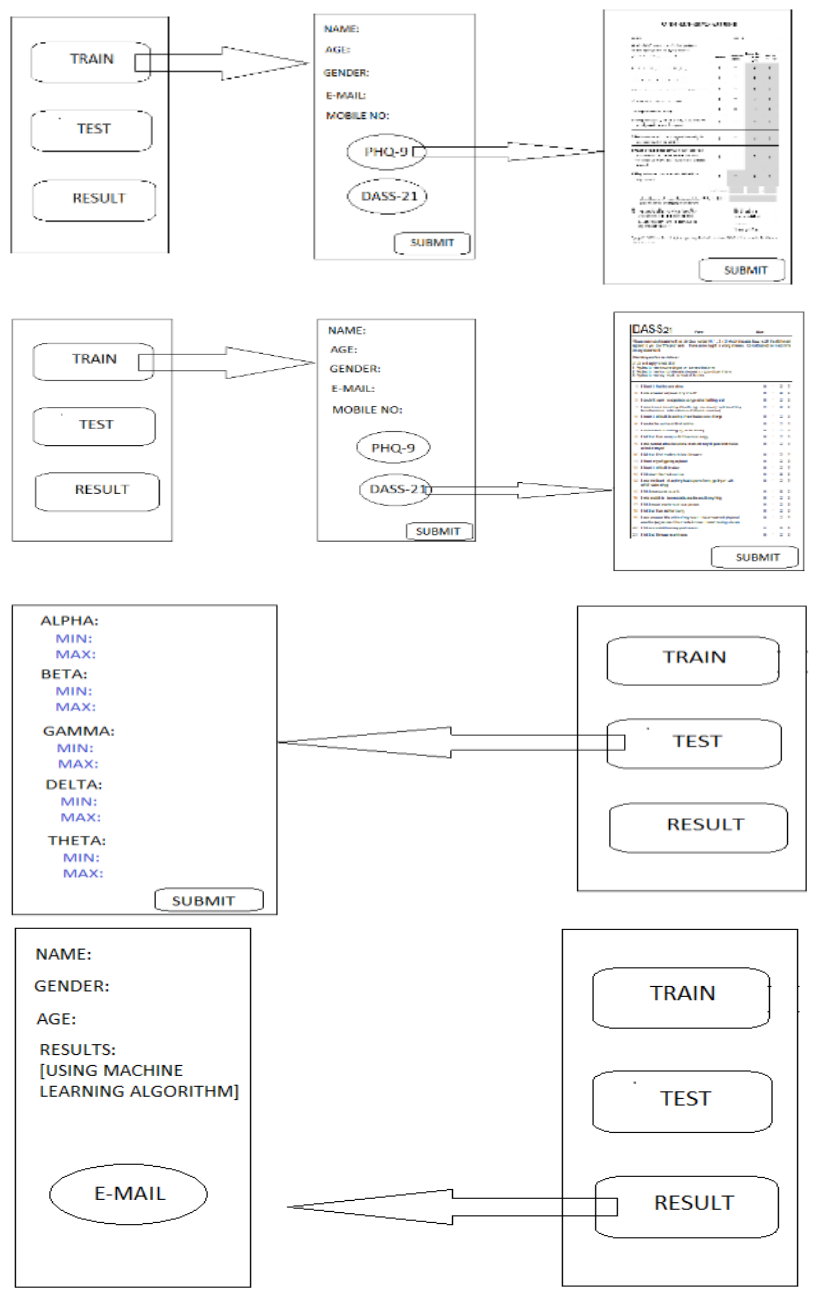

Fig. 2.Proposed Mobile App Architecture
The Depression level prediction will be done by collecting the EEG data signals from the brain by using the Neurosky's Mindwave Mobile Kit, which will give the raw EEG waves. It is a non-invasive method which uses only single electrode. Person will be asked with 10 questions from the standard PHQ.9 and 21 questions from Dass-21 questionnaires.

The Patient Health Questionnaire (PHQ) is a multiple-choice self-report register that is considered for diagnosing, monitoring and measuring the severity of depression.

The Depression, Anxiety and Stress Scale - 21 Items (DASS-21) is defined as a set of three self-report scales developed to quantify the emotional states of depression, apprehension and pressure.

Depending on the level of depression, the different EEG waves, namely Alpha, Beta, Theta, Delta and Gamma waves varies which are taken and saved by Lucid Scribe and are fed to the Machine Learner. Here, in the proposed module, the EEG signals of the subjects will be obtained by interviewing different age group subjects and determine their depression levels. The features will then be obtained from the EEG frequency bands. Data set of $\mathrm{N}$ number of subjects will be prepared and fed to the Machine Learner to detect depression level wherein N1 samples will be trained and N-N1 samples would be tested to evaluate Machine Learning.

The important milestones of this project are as follows:

- The subjects are asked questions from standard PHQ-9 and DASS-21 questionnaires and collection of EEG signal data from the subjects by using Neurosky's Mindwave Kit.

- Extraction and exporting the collected parameter from raw data.

- Statistical computation of the required data of each subject and creation of a new data feature set of all $\mathrm{N}$ subjects which are fed to the machine Learner.

- Classification of the set of test data of parameters to identify the depression level of the subject by using Machine Learner.

- Mobile App architecture is planned as shown in the Fig 2 to automate the data collection and reporting.

\section{CONCLUSION}

This work presents a predictor model method with advantages and limitations. The Predictor model may be designed with advanced machine learning algorithms. To improve the design portability, proposed App may be designed for the same.

\section{A. Advantages}

The Advantages of the proposed work are as follows.

- Successful diagnosis alongside current technology can confirm these objectives and help the experts in early detection and treatment.

- Due to its ease of use of proposed model of the app this technology can offer a visible tool for mental health professionals to identify symptoms of depression, thus enabling a faster preventive intervention. 
- The betterment of effective ML and sensing methods, computer based analysis plays an important role in giving a higher accurate result.

- Capable of detecting depression with minimal human intervention.

- This investigation might be a supporting guide for therapists to recognize the level of depression in patients.

\section{B. Limitations}

The Proposed work will be having few limitations which are listed below.

- Inappropriate diagnosis of the mental health using this technique could lead to incorrect treatment.

- Farmers sometimes find the measuring device to be a little intimidating in appearance and hence sometimes are apprehensive towards wearing it on their head.

- Since machine learning algorithm is been planned to develop, the predictor model requires 200 data samples which may not be sufficient to achieve an accuracy of $100 \%$.

- More number of software's are involved, hence expertise is required to validate.

\section{Social impact of the proposed work}

This work will enable one to use machine learning to predict depression level of a farmer and saves lives for the same.

\section{ACKNOWLEDGMENT}

The Authors thank the management of RNSIT- Dr. H N Shivashankar, Director, Dr. M K Venkatesha, Principal, Dr. G T Raju, Vice Principal, Dr. Andhe Pallavi, HoD, EIE Dept. for their ethical guidance.

\section{REFERENCES}

1. Shamla Mantri, Dr. Pankaj Agrawal, et. al., "Non Invasive EEG Signal Processing Framework for Real Time Depression Analysis", SAI Intelligent Systems Conference 2015 November 10-11, 2015, London. pp: 518-521

2. Xiaowei Li, Zhuang Jing, Bin Hu, "An EEG-based study on coherence and brain networks in mild depression cognitive process", Shuting Sun School of Information Science \& Engineering, Lanzhou University Lanzhou, China , Conference Paper . December 2016. DOI: 10.1109/BIBM.2016.7822702

3. Dr. G. L. Parvathamma, "Farmers Suicide and Response of the Government in India -An Analysis", IOSR Journal of Economics and Finance, Volume 7, Issue 3. Ver. 1. 2016, pp: 1-1.

4. Sharifa Alghowinem, Roland Goecke, et. al, "Multimodal Depression Detection: Fusion Analysis of Paralinguistic, Head Pose and Eye Gaze Behaviors", IEEE Transactions on Affective Computing, January 2015.

5. Arkaprabha Sau, Ishita Bhakta, "Predicting anxiety and depression in elderly patients using machine learning technology", Published in Healthcare Technology Letter" July 2017.

6. Mojtaba Hajian; Mohammad Hassan Moradi, "Quantification of Depression Disorder Using EEG Signal", 24th National and 2nd International Iranian Conference on Biomedical Engineering (ICBME). 2017. DOI: 10.1109/ICBME.2017.8430237

7. Yashika Katyal ; Suhas V Alur; Shipra Dwivedi ; Menaka R, "EEG signal and video analysis based Depression indication", IEEE International Conference on Advanced Communications, Control and Computing technologies. 2014

8. Yousef Mohammadi, Mojtaba Hajian, Mohammad Hassan Moradi, "Discrimination of Depression Levels Using Machine Learning Methods on EEG Signals", 27th Iranian Conference on Electrical Engineering (ICEE). Pp:1-8
9. H M Mallikarjun, H N Suresh.” Depression level prediction using EEG signal processing", 2014 International Conference on Contemporary Computing and Informatics (IC3I), 2014 DOI: 10.1109/IC3I.2014.7019674

\section{AUTHORS PROFILE}

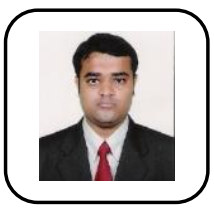

Dr. Mallikarjun $\mathbf{H}$ M is working in Electronic \& Instrumentation Engg department, RNSIT, Bengaluru. His area of interest is on BioSignals. He published his research works in various conferences/journals on emotion/Mental state, Sleep disorders and drunkenness classification by using brain signals.

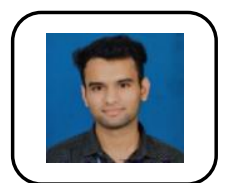

Akshay Chhetri, BE in Electronics and Instrumentation Engg. His area of interest includes Biomedical Engg, Aeronautical Instrumentation and Embedded Controllers.

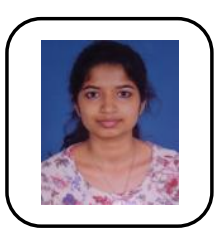

Apoorva G S, BE in Electronics and Instrumentation Engg. Her area of interest includes Programming, Biomedical Signal Processing and Embedded Controllers.

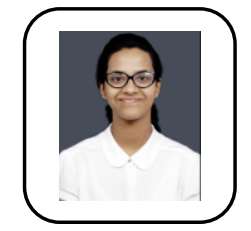

Gowri Jadhav , BE in Electronics and Instrumentation Engg. Her area of interest includes Aeronautical Instrumentation, Biomedical Instrumentation.

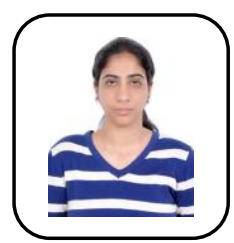

Sheetal B V, BE in Electronics and Instrumentation Engg. Her area of interest includes Biomedical Signal Processing, and Advanced Embedded Controllers. 